\title{
Genetic characterization of a novel Tib-derived variant of soybean Kunitz trypsin inhibitor detected in wild soybean (Glycine soja)
}

\author{
Ke-Jing Wang, Tetsuro Yamashita, Masao Watanabe, and Yoshihito Takahata
}

\begin{abstract}
A novel variant of soybean Kunitz trypsin inhibitor (SKTI) was detected in 530 lines of wild soybean (Glycine soja). This variant showed an intermediate electrophoretic mobility between the Tia and Tic types. In isoelectric focusing polyacrylamide gel electrophoresis gels containing urea, this variant had a similar isoelectric point as that of Tia. The genetic analysis of SKTI bands in $\mathrm{F}_{2}$ seeds from crosses of the new variant type with Tia or Tic type showed that this variant type is controlled by a codominant allele at the SKTI locus. We propose the genetic symbol Tif for this novel variant. When the nucleotide sequence of the Tif gene was compared with those of other types of SKTI genes (Tia, Tib, and Tic), the sequence of Tif was identical to that of Tib with the exception of one A $\rightarrow \mathrm{G}$ transitional mutation occurring at position 676 of Tif. This mutation resulted in an amino acid change from Lys to Glu at the 178 residue. These results suggest that this variant is derived from Tib through a point mutation. In addition, we settled an inconsistency in the number of amino acid differences between Tia and Tib (eight or nine). Analysis of nucleotide and amino acid sequences revealed that Tib was different from Tia by nine amino acids.
\end{abstract}

Key words: soybean Kunitz trypsin inhibitor, polymorphism, gene sequence, soybean, wild soybean.

\begin{abstract}
Résumé : Un nouveau variant de l'inhibiteur de trypsine de type Kunitz chez le soja (SKTI) a été détecté parmi 530 lignées de soja sauvage (Glycine soja). Ce variant présente une mobilité électrophorétique intermédiaire entre les types Tia et Tic. Sur des gels PAGE à focalisation isoélectrique contenant de l'urée, ce variant montre un point isoélectrique semblable à celui de Tia. Une analyse génétique des bandes SKTI au sein d'une population de graines $\mathrm{F}_{2}$ issues du croisement entre le nouveau variant et les types Tia ou Tic a révélé que ce variant est contrôlé par un allèle codominant au locus SKTI. Les auteurs proposent le symbole Tif pour ce nouveau variant. En comparant la séquence nucléotidique du gène Tif à celle des autres gènes SKTI (Tia, Tib et Tic), il a été observé qu'une seule transition A $\rightarrow \mathrm{G}$, à la position 676 du gène Tif, distingue ce dernier de la séquence du gène Tib. Cette mutation entraîne un changement d'acide aminé (Lys $\rightarrow$ Glu) au résidu 178. Ces résultats suggèrent que ce variant est survenu suite à une mutation ponctuelle dans le gène Tib. Des plus, les auteurs ont levé l'incertitude quant au nombre de différences qui distinguent Tia de Tib (huit ou neuf). L'analyse des séquences nucléotidiques et peptidiques a révélé que Tib diffère de Tia au niveau de neuf acides aminés.
\end{abstract}

Mots clés : inhibiteur de la trypsine de type Kunitz chez le soja, polymorphisme, séquence nucléotidique, soja, soja sauvage.

[Traduit par la Rédaction]

\section{Introduction}

Proteinase inhibitors are extensively contained in many plants and are considered to be involved in the control of endogenous proteinases, storage proteins, and defence against insect and microorganism damage (Ryan 1981; Richardson 1977; Johnson et al. 1989; Marchetti et al. 2000). The total seed protein of soybean (Glycine max) contains about 6\% proteinase inhibitors. These inhibitors have been identified as belonging to two major groups, the Kunitz trypsin inhibitor (Kunitz 1945) and the Bowman-Birk trypsin inhibitor (Bowman 1946; Birk 1961). Of these, the soybean Kunitz trypsin inhibitor (SKTI) has been found to have six electrophoretic distinguishable forms: Tia and Tib (Singh et al.

Received 24 March 2003. Accepted 21 July 2003. Published on the NRC Research Press Web site at http://genome.nrc.ca on 22 December 2003.

Corresponding Editor: F. Belzile.

K.-J. Wang. Faculty of Agriculture, Iwate University, Morioka 020-8550, Japan, and Institute of Crop Germplasm Resources, Chinese Academy of Agricultural Sciences, Beijing 100081, China.

T. Yamashita, M. Watanabe, and Y. Takahata. ${ }^{1}$ Faculty of Agriculture, Iwate University, Morioka 020-8550, Japan.

${ }^{1}$ Corresponding author (e-mail: ytakahata@iwate-u.ac.jp). 
Fig. 1. Identification of a novel variant of SKTI proteins by (A) a Davis system of PAGE and (B) Western blot analysis. Lane C, control of commercial SKTI (Tia) from Sigma; lane 1, Tic type ('Raiden'); lane 2, a novel variant of SKTI of $G$. soja; lane 3, Tia ('Rikuu No.27'); lane 4, Tib ('Tachisuzunari').

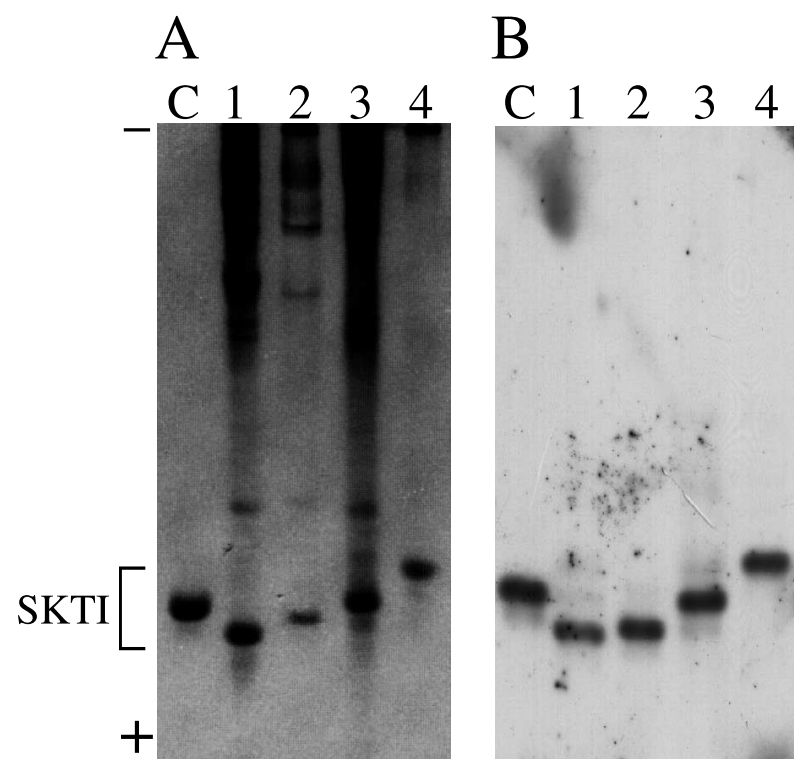

1969), Tic (Hymowitz 1973), Tid (Zhao and Wang 1992), Tie (Wang et al. 1996, 2001), and ti (null type) (Orf and Hymowitz 1979). These types are controlled by codominant multiple alleles at a single locus (Singh et al. 1969; Hymowitz and Hadley 1972; Orf and Hymowitz 1977; Wang et al. 2001). The polymorphism of SKTI has been widely used for the research of genetic diversity, dissemination, and origin of soybean (Hymowitz and Kaizuma 1979; Kaizuma et al. 1980; Yu and Kiang 1993; Xu et al. 1985).

Kaizuma et al. (1980) considered that the Tia type is the prototype from which Tib and Tic were derived. Studies of amino acid and nucleotide sequences revealed that Tic, Tid, and Tie each were originally generated from the prototype Tia by a single amino acid substitution (Kim et al. 1985; Jofuku et al. 1989; Song et al. 1993; Xin et al. 1999; Wang et al. 2001). The Tib type has also been considered to be a secondary ancient type that arose from Tia before the domestication of cultivated soybean from wild soybean (Glycine soja) (Kaizuma et al. 1980). If Tib is a second prototype, it is probable that some variant types derived from Tib are still in existence. However, so far, such variants have not been found.

Since Kim et al. (1985) reported a comparative study of the amino acid sequences of SKTI, it has been believed that Tib differed from Tia by eight amino acid residues. In contrast, a study of nucleotide sequences showed a difference of nine amino acids between them (Song et al. 1993). Therefore, a reanalysis is needed to account for this inconsistency. There are such large sequence differences between Tia and Tib, but no intermediate types between them have been found in either cultivated or wild soybean.

In the present report, we describe detection and genetic characterization of a novel polymorphic variant (designated as Tif) of SKTI derived from the Tib type in wild soybean
Fig. 2. Identification of a novel variant of SKTI proteins by (A and B) IEF-PAGE and (C and D) Western blot analysis (A and C) containing $8 \mathrm{M}$ urea and (B and D) without urea. Lane 1, Tic type ('Raiden'); lane 2, a novel variant of SKTI of G. soja; lane 3, Tia ('Rikuu No. 27'); lane 4, Tib ('Tachisuzunari').

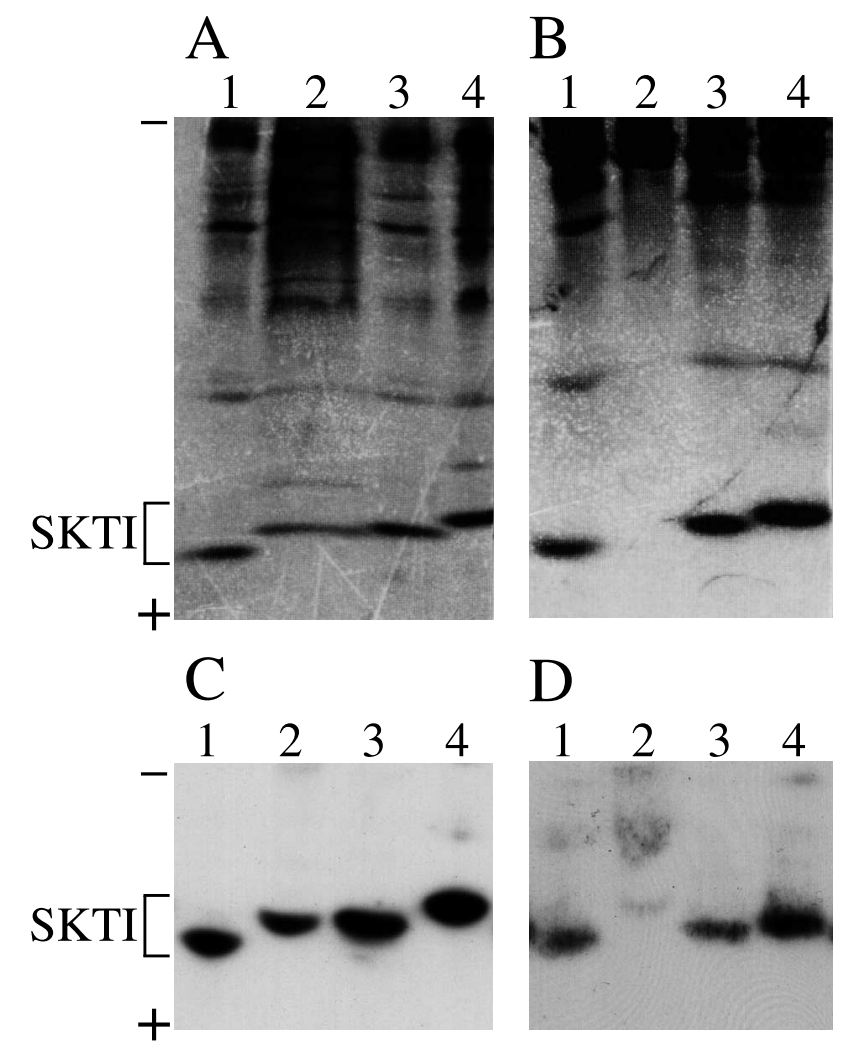

and settle the discrepancy in the number of amino acids that are different between Tia and Tib.

\section{Materials and methods}

\section{Plant materials}

In this investigation, 530 wild soybean (G. soja) lines collected in China were used to analyze the polymorphism of the SKTI proteins by electrophoresis. Soybean (G. max) cultivars or lines 'Rikuu No. 27' (Tia), 'MG-3' (Tia), 'Odate No. 1' (Tib), 'Norin No. 2' (Tib), 'Tachisuzunari' (Tib), 'Raiden' (Tic), and 'L-188-21' (Tic) and a wild soybean line 74 (Tib) collected in China were used as materials of standard SKTI proteins.

\section{Electrophoretic detection}

Extraction of SKTI proteins from seeds was carried out according to Hymowitz and Hadley (1972). The SKTI proteins were analyzed by a Davis system of polyacrylamide gel electrophoresis (PAGE) and isoelectric focusing (IEF)-PAGE as described previously (Wang et al. 1996) with minor modifications. IEF-PAGE was performed using a gel containing $1.2 \%$ pharmalyte ( $\mathrm{pH} 4-6.5$ ) and $0.4 \%$ pharmalyte $(\mathrm{pH} 3-10)$ with or without $8 \mathrm{M}$ urea.

After electrophoresis, the proteins were electroblotted onto a Immobilon-P membrane (Millipore) for Western blot analysis. SKTI proteins were detected using anti-trypsine in- 
Table 1. SKTI band segregation of $\mathrm{F}_{2}$ seeds from the crosses of a new variant (Tif) with Tia and Tic types.

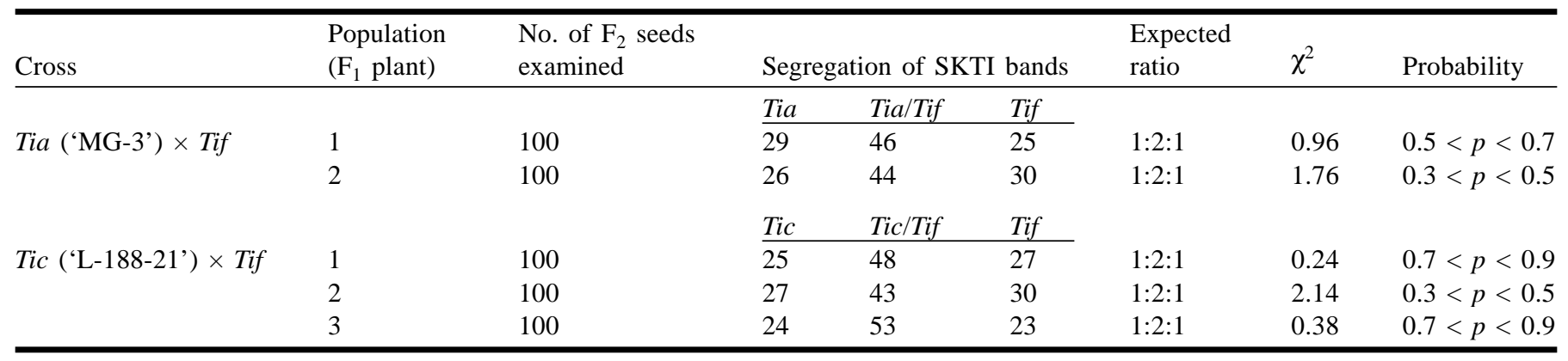

Fig. 3. Segregation of the Tif variant in $\mathrm{F}_{2}$ derived from $\mathrm{F}_{1}$ between Tif and Tic ('Raiden'). Lane 1, Tif type; lanes 2 and 3, hetero type having both Tif and Tic; lane 4, Tic type.

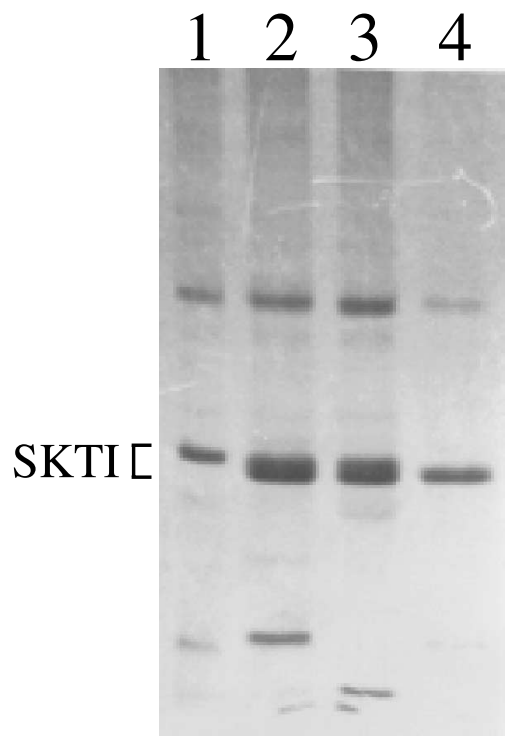

hibitor (soybean) rabbit antiserum (Rockland) and ECL Western Blotting Detection Regents Kit (Amersham Biosciences).

\section{Genetic analysis}

The new variant line of SKTI (Tif) proteins found in this study was crossed with the G. max 'MG-3' (Tia) and 'L-188-21' (Tic) lines in a growth chamber. The $\mathrm{F}_{1}$ plants of these crosses were grown in an experimental field to obtain $\mathrm{F}_{2}$ seeds, and SKTI of the $\mathrm{F}_{2}$ seeds was examined electrophoretically.

\section{$\mathrm{N}$-terminal amino acid sequence analysis}

SKTI proteins were separated by IEF-PAGE and electrotransferred to the polyvinylidene difluoride membrane, and the N-terminal amino acid sequences of the blotted proteins were determined using a protein sequencer (PPSQ-21, SHIMADZU).

\section{Gene sequence analysis}

Total DNA was extracted from a single seed according to McDonald et al. (1994), Tian et al. (2000), and Wu et al. (2001) with minor modifications. The SKTI gene was amplified by polymerase chain reaction using a set of two primers (forward: 5'-TAGTCCCGATTCTCCCAACA-3', reverse: 5'-AGTACTCTCACACTTGTGTC-3') (Wang et al. 2001). After the amplified DNA was cloned using a TA Cloning Kit (Invitrogen) according to Wang et al. (2001), it was sequenced with an ABI Prism 310 sequencer with a BigDye Terminator Cycle Sequencing Kit (Applied Biosystems). Two independent clones from each of the two seeds were analyzed in each line.

\section{Results and discussion}

\section{Detection of a novel SKTI type (Tif) in wild soybean}

Almost all SKTI electrophoretic mobility types of the 530 wild soybean showed Tia (85\%) and Tib (15\%) types, except for one line. This variant line (line 33) showed a novel SKTI electrophoretic mobility that is intermediate between those of Tia and Tic (Fig. 1). The variant was also compared with three standard SKTI types (Tia, Tib, and Tic) using IEF-PAGE. The different banding patterns of these types in IEF-PAGE gel are shown in Fig. 2. In the presence of $8 \mathrm{M}$ urea, this variant was definitely distinguished from $T i b$ and Tic by its isoelectric point, having almost a similar isoelectric point as that of Tia (Figs. 2A and 2C). This variant has a feature different from other types of Tia, Tib, and Tic in the IEF system. This variant could not be recognized in the IEF gel without urea because of its disappearing band (Figs. 2B and 2D, lane 2). The exact reason why the band of the variant disappeared in the IEF gel without urea is unknown. One possibility is that the novel SKTI may shape the aggregate form after proteins are extracted. However, to elucidate the mechanism of this phenomenon, further investigation is needed.

To investigate the genetic stability of the variant, the variant plant was grown and the selfed seeds were harvested. The electrophoretic results of the SKTI proteins from the selfed seeds showed that the offspring of the variant had the same mobility band, suggesting that the SKTI type of this variant was an inheritable trait (data not shown). We designated Tif as the genetic symbol for this novel variant type. Two populations of $\mathrm{F}_{2}$ seeds obtained from two $\mathrm{F}_{1}$ s between Tia ('MG-3') and Tif variant and three populations of $\mathrm{F}_{2}$ seeds obtained from three $\mathrm{F}_{1}$ s between Tic ('L-188-21') and Tif were individually analyzed by PAGE for the segregation ratio of the new SKTI type. The genetic segregation ratio of the $\mathrm{F}_{2}$ seeds in the two crosses showed an acceptable fit to a 1:2:1 ratio of the Tia (or Tic) band to both the Tia (or Tic) and the Tif bands to the Tif band (Table 1; Fig. 3). These re- 
Fig. 4. Nucleotide and deduced amino acid sequences of the SKTI genes of Tia ('Rikuu No. 27') (DDBJ accession No. AB112031), Tic ('Raiden') (DDBJ accession No. AB112033), Tib ('Odate No. 1', 'Tachisuzunari', 'Norin No. 2', and G. soja line 74) (DDBJ accession No. AB112032), and Tif (a novel variant of G. soja) (DDBJ accession No. AB112034). Dashes in sequences indicate identical nucleotides and amino acids. The box shows the single amino acid of Tif that differed from Tib. Horizontal arrows show the positions of primers used to amplify the sequences. The mature SKTI proteins begin at $\mathrm{D}^{*}$ and end at $\mathrm{L}^{*}$.

Table 2. Comparison of the beginning 14 amino acid sequences at the $\mathrm{N}$ terminus among different SKTI types.

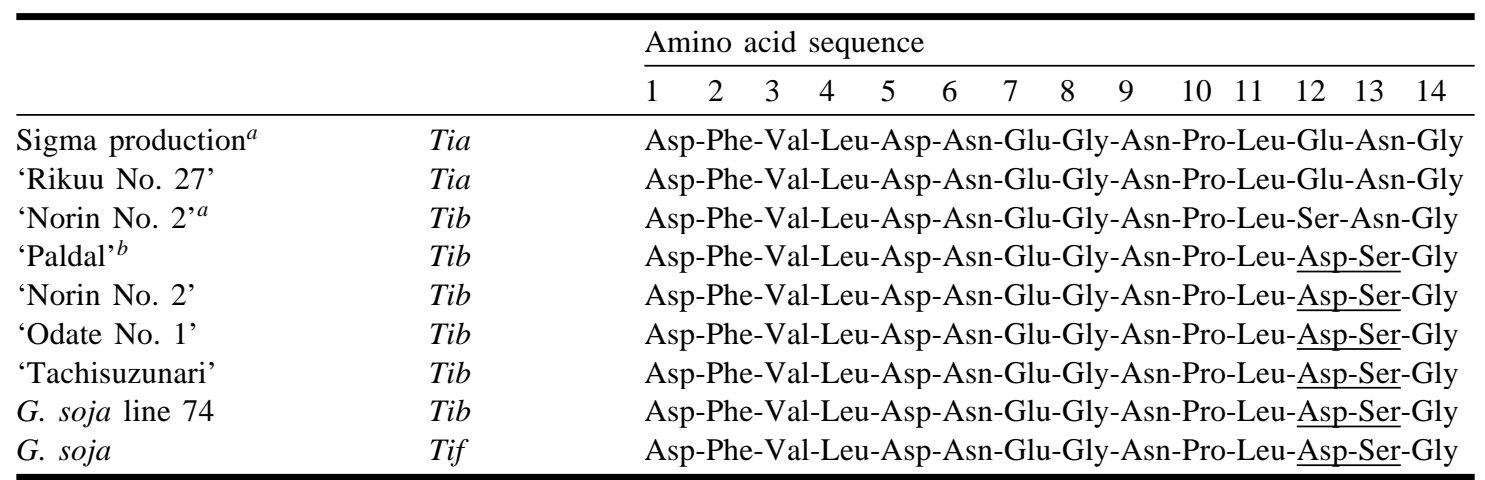

\footnotetext{
Note: The sequences were determined for the protein bands in an IEF-PAGE gel. Underscoring indicates the correct amino acid sequences at residues 12 and 13 in Tib.

${ }^{a}$ Sequences quoted from the results reported by Kim et al. (1985).

${ }^{b}$ Sequences deduced from the nucleotide sequences reported by Song et al. (1993).
}

sults demonstrate that Tif is a genetically new codominant allele at the SKTI locus.

Nucleotide sequence analysis indicated that all of the amplified fragments had an identical length of $743 \mathrm{bp}$. The sequences contained an open reading frame of $651 \mathrm{bp}$ encoding 271 amino acids, which consisted of 181 amino acids of a mature SKTI, signal regions of 25 amino acids at the $\mathrm{N}$ terminus, and 11 amino acids at the $\mathrm{C}$ terminus (Fig. 4). Such extra amino acids at the $\mathrm{N}$ terminus and $\mathrm{C}$ terminus were reported by Song et al. (1993) and Wang et al. (2001). The sequences of Tia and Tib were identified with those reported by Song et al. (1993). Comparing the sequence of the Tif gene with other types of SKTI, the sequences of Tif and Tib were identical except for one A $\rightarrow \mathrm{G}$ transitional mutation that occurred at position 676 of Tif (Fig. 4). This mutation resulted in an amino acid change from Lys to Glu at the 178 residue. To date, the known SKTI types, such as Tic (Kim et al. 1985), Tid (Xin et al. 1999), and Tie (Wang et al. 2001), have been shown to differ from Tia in a single amino acid residue, suggesting that these SKTI types originated from the Tia type by point mutation. Our results indicate that the novel SKTI type of Tif originated from Tib. This was supported by the existence of the same silent mutation at position 459 in both Tib and Tif (Fig. 4). Namely, the sequence of the Tib gene differed from Tia and Tic by a $\mathrm{T} \rightarrow \mathrm{C}$ mutation at position 459 , which does not cause amino acid change (Tia and Tic: GTT, Tib: GTC). In common with Tib, the Tif gene had the identical mutation at the same position.

\section{Comparisons of amino acids between Tia and Tib}

There is inconsistency in the number of amino acid differences between Tia and Tib SKTI proteins. Kim et al. (1985) reported that the Tib type differs from Tia by the following eight amino acid residues based on amino acid sequence analysis: Ser (12), Phe (62), Asn (71), Arg (74), Val (114), Ile (120), Thr (137), and Val (176); in contrast, Song et al. (1993) demonstrated that Tib differed from Tia by the following nine amino acids based on nucleotide sequence analysis: Asp (12), Ser (13), Phe (62), Asn (71), Arg (74), Val (114), Ile (120), Thr (137), and Val (176). The difference between them is present at the 12th and 13th amino acid residues of the mature SKTI protein: Ser (12) - Asn (13) ('Norin No. 2'; Kim et al. 1985) and Asp (12) - Ser (13) ('Paldal'; Song et al. 1993). The possibility remained that such disagreement may be due to the difference in cultivars used. We analyzed the nucleotide sequences of the SKTI genes in four kinds of $\mathrm{Tib}$ materials ('Norin No. 2', 'Odate No. 1', 'Tachisuzunari', and G. soja line 74) and one Tia ('Rikuu No. 27'). Of these Tib materials analyzed, 'Norin No. 2' is the same cultivar as the one used by Kim et al. (1985). Our results showed that all four Tib materials had the same nucleotide sequences, and their deduced amino acids 12 and 13 are Asp-Ser rather than Ser-Asn, suggesting that there were nine amino acid differences between Tia and Tib and not eight (Fig. 4). This is supported by the results from the sequence analysis of amino acids at the $\mathrm{N}$ terminus of the mature SKTI proteins, which revealed that two amino acids were different between Tib and Tia at this region (Table 2). These results support those of Song et al. (1993) rather than those of Kim et al. (1985). It is thus concluded that Tib is different from Tia by nine amino acid residues.

The nucleotide sequence of the Tic gene ('Raiden'), which was first determined in this study, is identical to the sequence of Tia except for one $\mathrm{G} \rightarrow \mathrm{A}$ transition occurring at position 308 of Tic (Fig. 4). This mutation results in an 


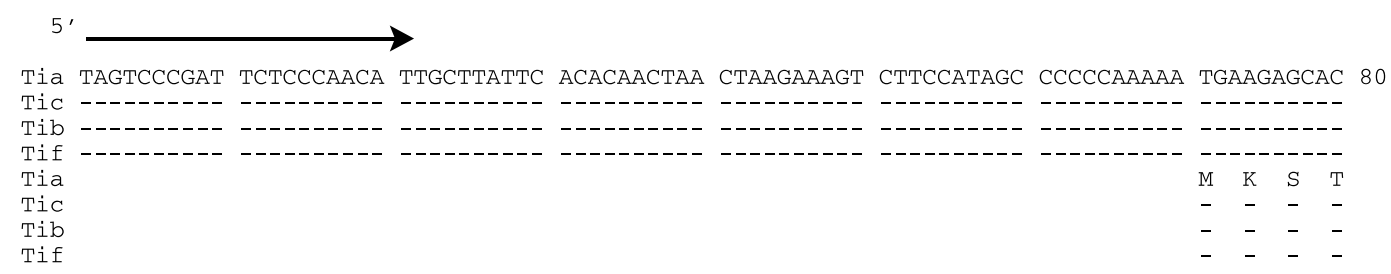

CATCTTCTTT GCTCTCTTTC TCTTTTGTGC CTTCACCACC TCATACCTAC CTTCAGCCAT CGCTGATTTC GTGCTCGATA 160

\begin{tabular}{|c|c|c|c|c|c|c|c|c|c|c|c|c|c|c|c|c|c|c|c|c|c|c|c|}
\hline & & & & & & & & & & & & & & & & & & & & & & & \\
\hline & & & A & L & $\mathrm{F}$ & L & $\mathrm{F}$ & C & A & $\mathrm{F}$ & T & $\mathrm{T}$ & $S$ & $\mathrm{Y}$ & L & P & S & A & I & A & $D^{*}$ & $F$ & L \\
\hline & - & 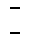 & - & - & - & - & - & - & - & - & - & - & - & - & - & - & - & - & - & - & - & - & - \\
\hline & & & - & - & & - & - & & & - & & & & & & & & & & & & & \\
\hline
\end{tabular}

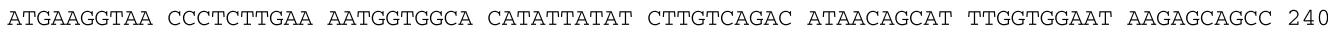

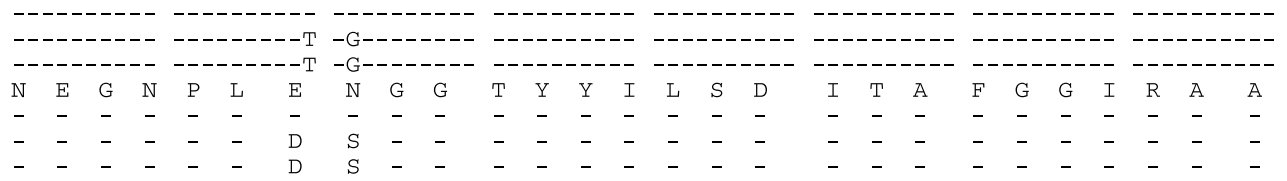

CCAACGGGAA ATGAAAGATG CCCTCTCACT GTGGTGCAAT CTCGCAATGA GCTCGACAAA GGGATTGGAA CAATCATCTC 320

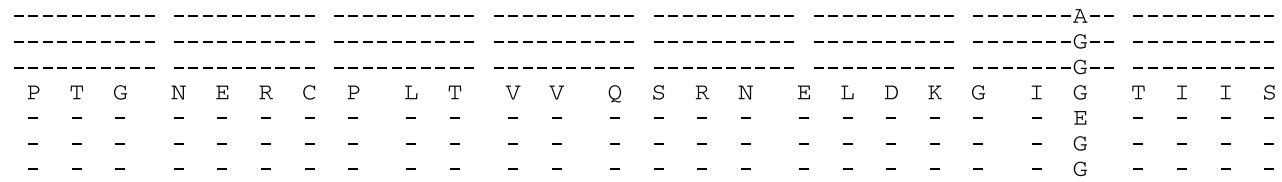

GTCCCCATAT CGAATCCGTT TTATCGCCGA AGGCCATCCT TTGAGCCTTA AGTTCGATTC ATTTGCAGTT ATAATGCTGT 400 $\begin{array}{cccccccccccccccccc}- & \end{array}$

GTGTTGGAAT TCCTACCGAG TGGTCTGTTG TGGAGGATCT ACCAGAAGGA CCTGCTGTTA AAATTGGTGA GAACAAAGAT 480

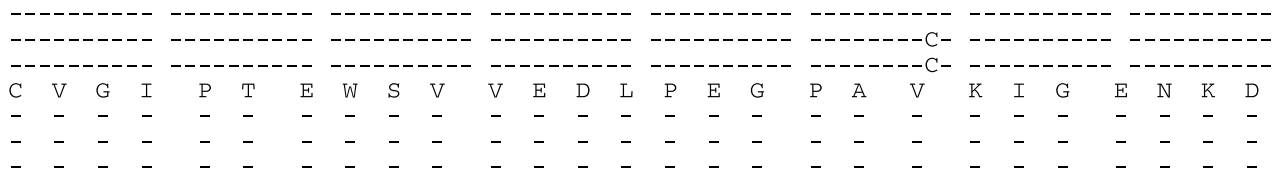

GCAATGGATG GTTGGTTTAG ACTTGAGAgA GTTTCTGATG ATGAATTCAA TAACTATAAG CTTGTGTTCT GTCCACAGCA 560

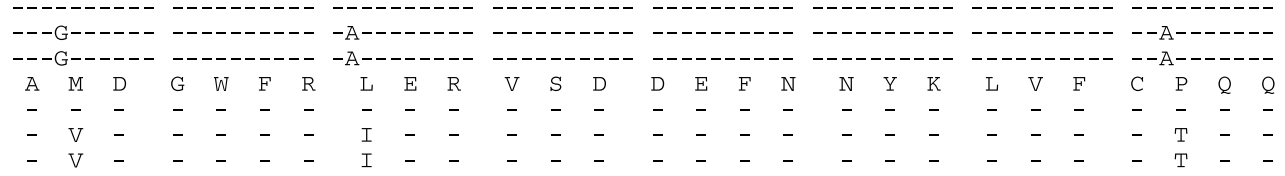

AGCTGAGGAT GACAAATGTG GGGATATTGG GATTAGTATT GATCATGATG ATGGAACCAG GCGTTTGGTG GTGTCTAAGA 640

$\begin{array}{cccccccccccccccc}- & \text { A }\end{array}$

ACAAACCGTT AGTGGTTCAG TTTCAAAAAC TTGATAAAGA ATCACTGGCC AAGAAAAATC ATGGCCTTTC TCGCAGTGAg 720

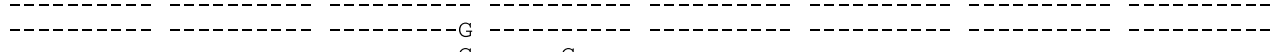
$\begin{array}{llllllllllllllllllllllllllllllllll}N & K & P & L & V & V & Q & F & Q & K & L & D & K & E & S & L^{*} & A & K & K & N & H & G & L & S & R & S & E\end{array}$ \begin{tabular}{lllllllllllllllllllllllllll}
$N$ & - & - & $L$ & - & - & - & - & - & $K$ & $L$ & $D$ & $K$ & $E$ & $S$ & $L$ & $A$ & $K$ & $K$ & $N$ & $H$ & $G$ & $L$ & $S$ & $R$ & $S$ & $E$ \\
- & - & - & - & - & - & - & - & - & - & - & - & - & - & - & - & - & - & - & - & - & - & - & - & - & - & - \\
- & - & - & - & - & - & - & - & - & - & $V$ & - & - & - & - & - & - & - & - & - & - & - & - & - & - & - & - \\
\hline
\end{tabular} TGAGACACAA GTGTGAGAGT ACT 743

(-10

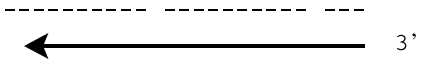


amino acid change from Gly to Glu at the 55 residue. This result coincides with the amino acid sequence of Tic ('Raiden') revealed by Kim et al. (1985).

In this study, we discovered a novel Tib-derived Tif variant that is one of the multiple alleles at the SKTI locus. This is the first report showing a variant of SKTI originating from Tib. In addition, we determined a sequence difference in nine amino acid residues between Tia and Tib. Thus far, of several SKTI forms, Tia, Tib, and Tic have been detected in both wild and cultivated soybean, Tid was found in cultivated soybean (Zhao and Wang 1992), and Tie was observed in wild soybean (Wang et al. 1996, 2001). Since Tic, Tid, and Tie each differ from Tia by one amino acid, these types are considered to originate from prototype Tia by a point mutation. The Tif variant detected in this study differs from Tib by one amino acid, showing a novel variant that originated from Tib. Kaizuma et al. (1980) considered that the Tia type is the prototype from which Tib and Tic were derived and that differentiation of Tib from Tia occurred much earlier than that of Tic from Tia. Our results support their idea that Tib is a secondary ancient type of SKTI. On the other hand, there are large differences in nine amino acid residues between Tia and Tib. Thus far, no intermediate type between them has been found in either soybean or wild soybean. A search of such an intermediate variant is currently being carried out.

\section{Acknowledgements}

We wish to thank Professor Emeritus N. Kaizuma for his encouragement and advice. We also thank Professor H. Taira for his useful suggestions. This work was supported by a Grant-in-Aid for Scientific Research from the Ministry of Education, Science, Culture, and Sports, Japan.

\section{References}

Birk, Y. 1961. Purification and some properties of a highly active inhibitor of trypsin and $\alpha$-chymotrypsin from soybean. Biochim. Biophys. Acta, 54: 378-381.

Bowman, D.E. 1946. Differentiation of soy bean antitryptic factors. Proc. Soc. Exp. Biol. Med. 63: 547-550.

Hymowitz, T. 1973. Electrophoretic analysis of SBTI-A2 in the USDA soybean germplasm collection. Crop Sci. 13: 420-421.

Hymowitz, T., and Hadley, H.H. 1972. Inheritance of a trypsin inhibitor variant in seed protein of soybean. Crop Sci. 12: 197-198.

Hymowitz, T., and Kaizuma, N. 1979. Dissemination of soybean (Glycine max): seed protein electrophoresis profiles among Japanese cultivars. Econ. Bot. 33: 311-319.

Jofuku, K.D., Schipper, R.D., and Goldberg, R.B. 1989. A frameshift mutation prevents Kunitz trypsin inhibitor mRNA accumulation in soybean embryos. Plant Cell, 1: 427-435.

Johnson, R., Narvaez, J., An, G., and Ryan, C. 1989. Expression of proteinase inhibitors I and II in transgenic tobacco plants: effects on natural defense against Manduca sexta larvae. Proc. Natl. Acad. Sci. U.S.A. 86: 9871-9875.

Kaizuma, N., Oikawa, K., and Miura, M. 1980. Consideration on the cause of the differential $\mathrm{Ti}$ alleles frequency distributions found among some regional populations of soybean (Glycine $\max ($ L.) Merrill) land varieties. J. Fac. Agric. Iwate Univ. 15: 81-96.

Kim, S.H., Hara, S., Hase, S., Ikenaka, T., Tode, H., Kitamura, K., and Kaizuma, N. 1985. Comparative study on amino acid sequence of Kunitz-type soybean trypsin inhibitors, Tia, Tib, and Tic. J. Biochem. 19: 435-448.

Kunitz, M. 1945. Crystallization of trypsin inhibitor from soybean. Science (Wash., D.C.), 101: 668-669.

Marchetti, S., Delledonne, M., Fogher, C., Chiaba, C., Chiesa, F., Savazzini, F., and Giordano, A. 2000. Soybean Kunitz, C-II and PI-IV inhibitor genes confer different levels of insect resistance to tobacco and potato transgenic plants. Theor. Appl. Genet. 101: 519-526.

McDonald, M.B., Elliot, L.J., and Sweeney, P.M. 1994. DNA extraction from dry seed for RAPD analyses in varietal identification studies. Seed Sci. Technol. 22: 171-176.

Orf, J.H., and Hymowitz, T. 1979. Inheritance of the absence of the Kunitz trypsin inhibitor in seed protein of soybeans. Crop Sci. 19: $107-109$.

Richardson, M. 1977. The proteinase inhibitor in plants and microorganisms. Phytochemistry, 16: 159-169.

Ryan, C.A. 1981. Proteinase inhibitors. In Biochemistry of plants. Vol. 6. Edited by A. Marcus. Academic Press, New York. pp. 351-370.

Singh, L., Wilson, C.M., and Hadley, H.H. 1969. Genetic differences in soybean trypsin inhibitor separated by disc electrophoresis. Crop Sci. 9: 489-491.

Song, S.I., Kim, C.H., Baek, S.J., and Choi, Y.D. 1993. Nucleotide sequences of cDNAs encoding the precursors for soybean (Glycine max) trypsin inhibitors (Kunitz type). Plant Physiol. 101: 1401-1402.

Tian, Q.Z., Gai, J.Y., Yu, D.Y., and Jia, J.Z. 2000. A study of amplified fragment length polymorphism (AFLP) in soybean. Soybean Sci. 19: 210-217.

Wang, K.J., Kaizuma, N., Takahata, Y., and Hatakeyama, S. 1996. Detection of two new variants of soybean Kunitz trypsin inhibitor through electrophoresis. Breeding Sci. 46: 39-44.

Wang, K.J., Takahata, Y., Ito, K., Zhao, Y.P., Tsutsumi, K., and Kaizuma, N. 2001. Genetic characterization of a novel soybean Kunitz trypsin inhibitor. Breeding Sci. 51: 185-190.

Wu, X.L., He, C.Y., Chen, S.Y., Zhuang, B.C., Wang, K.J., and Wang, X.C. 2001. Phylogenetic analysis of interspecies in genus Glycine through SSR markers. Acta Genet. Sin. 28: 359-366.

Xin, H., Xie, K.F., Dong, A.W., Yan, Q.Y., and Gu, Q.M. 1999. The amino acid sequence determination of a new variant of Kunitz soybean trypsin inhibitor (SBTi-A2). Soybean Genet. Newsl. [online]. Available from www.soygenetics.org/articles/ sgn.1999-004.html [posted 24 March 1999].

Xu, B., Zhao, S.W., Zou, S.H., Zheng, H.Y., Hu, Z.A., and Wang, H.X. 1985. Seed protein electrophoresis profiles of wild soybean ( $G$. soja) in China: the frequencies and geographical distribution of Tia and Sp1 alleles and the hypothesis on the original area of soybean. Soybean Sci. 4: 7-13.

Yu, H.Y., and Kiang, T. 1993. Genetic variation in South Korean natural populations of wild soybean (Glycine soja). Euphytica, 68: $213-221$.

Zhao, S.W., and Wang, H. 1992. A new electrophoretic variant of SBTi-A2 in soybean seed protein. Soybean Genet. Newsl. 19: 22-24. 